\title{
A Real-World Perspective on Interfacility Transfers of Acute Ischemic Stroke from a Semi-Rural Center
}

\author{
Lluís Llauger*, Ester Puyuelo, Francisco Sanchez-Mendez \\ Emergency Department, Vic University Hospital, Vic, Spain \\ Email: *llauger.doc@gmail.com
}

How to cite this paper: Llauger, L., Puyuelo, E. and Sanchez-Mendez, F. (2020) Paper Title. Open Journal of Emergency Medicine, 8, 1-12.

https://doi.org/10.4236/ojem.2020.81001

Received: January 8, 2020

Accepted: February 1, 2020

Published: February 4, 2020

Copyright $\odot 2020$ by author(s) and Scientific Research Publishing Inc. This work is licensed under the Creative Commons Attribution International License (CC BY 4.0).

http://creativecommons.org/licenses/by/4.0/

\begin{abstract}
Introduction: Interfacility transfers (IFT) of acute ischemic stroke (AIS) may not always lead to a better prognosis. Methods: Retrospective cohort study included AIS patients at an emergency department (ED) with telestroke. Multiple linear regression for departure time from ED (DT), quantile regression for length of in-hospital stay (LOS), and Kaplan-Meier estimator with Cox proportional hazards model for one-year survival (SV) were performed. Results: 192 patients included were categorised according to IFT. Mechanical thrombectomy was performed in $50 \%$ who had been transferred. Differences were found in DT, discharge disposition and LOS. An inverse relationship existed between DT and NIHSS. The strongest predictor of LOS was TACS ( $\beta$ $=3.14[0.03-8.49] ; \mathrm{p}=0.005)$. SV was related to IFT (HR 4.68 [1.37 - 16.07]; $\mathrm{p}=0.014)$, age (HR $1.1[1.04-1.17]), \mathrm{BI}<60$ (HR 2.7 [1.02 - 7.1]), TACS (HR 9.82 [1.08 - 88.95]) and NIHSS $\geq 6$ (HR 2.85 [1.05 - 7.74]). Conclusions: Shared decision-making with a stroke unit through telemedicine enabled a standardised clinical management in a non-metropolitan setting. Several improvement opportunities were identified: multimodal computed tomography availability before transfer, as well as optimization of response time and training in neurosonology of emergency physicians.
\end{abstract}

\section{Keywords}

Stroke, Emergency Room, Transportation of Patients, Prognosis

\section{Introduction}

Ischemic cerebrovascular disease (iCVD) represents a high-impact process in Spanish healthcare system. In the year 2017, stroke was the main diagnosis of 61,164 among $4,862,352$ hospital admissions; $78 \%$ aged $\geq 65$ years, a mean length 
of in-hospital stay (LOS) of 10.79 days and a mean cost of $€ 6137.64$ per admision [1]. In addition, iCVD was responsible for 7643 in-hospital deaths (1.8\% of total), $95 \%$ aged $\geq 65$ years ( $59 \%$ women) [2]. In the Autonomous Community of Catalonia, reperfusion strategy for acute ischemic stroke (AIS) was indicated in total for 3752 patients, exclusively in accordance with a standardised protocol by the health care administration [3].

An American study that analysed data for 2006-2014 period observed a 300\% increase in the probability of interfacility transfer (IFT) from ED, especially in patients from rural settings [4]. Mechanical thrombectomy of AIS involves a network of highly complex centers geostrategically located, often requiring an IFT. Sonig et al. [5] described that the hospitalisation costs of those transferred $(\$ 97,547)$ differed from those who were not $(\$ 70,325)$. Consequently, the increase in costs and variability between centres has generated the need to establish efficiency criteria. Therefore and with this objective, the implementation of telematic tools for specialised consultation [6] aims to increase diagnostic accuracy [7] and avoid transfers considered futile because so many times they do not involve the application of additional techniques by the neurologist.

Another common reason for IFT is transient ischemic attack (TIA). Some authors have published statistical models that predict the development of AIS after a short clinical event [8] [9], although additional neuroradiology techniques have been suggested to improve the discriminatory power of these scores [10], such as ultrasound of the supraaortic trunks (SAT) in the first 24 - 48 hours [11].

Therefore, we consider it necessary to explore IFT process of patients with AIS/TIA through a cohort of an unselected population, such as patients attended by emergency physicians in a semi-rural community hospital. The objective of this study was to describe clinical characteristics of patients with AIS/TIA, diagnostic process, and prognostic impact of IFT in order to identify opportunities to improve decision making.

\section{Methods}

We performed a retrospective cohort study, from January to December of 2017, in ED of Vic University Hospital. Patients consecutively included were aged $\geq 16$ years and diagnosed with AIS/TIA. False positives as well as those that lacked evolutive data were excluded. Evolutive follow-up was carried out by accessing shared medical history of the catalan healthcare system. Patients (or caregiver) must give their consent to be included in the study.

Cases were selected by reviewing the administrative record of past episodes coded as AIS or TIA. Medical charts are computerised in our center and hence variables defined in study protocol were recorded directly in an electronic database (Microsoft ${ }^{\oplus}$ Excel). The researchers included all episodes in which an acute neurological deficit was described, with a 24-hour limit for TIA. 35 independent variables were collected: 2 demographic (age and gender), 3 basal functional status and comorbidity scales (Barthel Index $<60[\mathrm{BI}]$, mRankin $\leq 1$, Charlson Index [CI]), 4 cardiovascular risk factors (smoke, hypertension, diabetes melli- 
tus, dyslipidemia), 5 comorbidities (cognitive impairment, atrial fibrillation [AF], mitral valvulopathy, peripheral arteriopathy, ischemic cardiopathy), 8 chronic medications (acetylsalicylic acid $100 \mathrm{mg}$, acetylsalicylic acid $300 \mathrm{mg}$, P2Y12 inhibitors, vitamin $\mathrm{K}$ inhibitors, direct oral anticoagulants, angiotensin II converting enzyme inhibitors, angiotensin II receptor antagonists, lipid-lowering drugs), 13 from the acute episode (type of access to ED [by own means, primary or prehospital or subacute healthcare], Oxfordshire/Bamford topographic diagnosis [LACS, PACS, POCS, TACS], AF + INR $<2$ seconds, time of onset of symptoms, wake-up stroke, NIHSS $\geq 6$, NIHSS $\geq 6+$ mRankin $\leq 1$, alteplase [rtPA], TIA SAT, AIS SAT, echocardiography, MRI). Multimodal CT (MCT), mechanical thrombectomy (MT) and admission to a stroke unit (SU) were registered only in transferred patients. Furthermore, 9 follow-up variables: DT from ED, discharge disposition (home, death, subacute healthcare center), ED reconsultation, LOS, 30-day death, 1-year death, 1-year death in NIHSS $\geq 6+$ mRankin $\leq 1$ group, 1 -year delta Barthel Index and mRankin in NIHSS $\geq 6$ group.

The study was undertaken following ethical principles for human research of Helsinki Declaration, and the protocol was approved by the Committee of Ethics and Clinical Investigation of Osona Foundation for Health Research and Education (CEIC code 2018978, own code PR209).

Quantitative variables were expressed as mean and standard deviation, if they followed a normal distribution, according to the Kolmogorov-Smirnov test, or median and interquartile range if not. Qualitative variables were described as absolute values and ratios.

The cohort was categorised according to IFT (TRANSFER, NON-TRANSFER). Only TRANSFER was sub-divided depending on NIHSS $\geq 6$, for the comparisons of MCT, MT and SU. Chi-squared, Fisher's exact and Cochran-Mantel-Haenszel tests, as appropriated, were applied to compare ratios. For quantitative variables, the tests applied were the Student's t (two means), if a normal distribution was followed, or Wilcoxon-Mann-Whitney (two medians) alternatively. Results were assumed to be significant with a p-value $<0.05$.

For multivariate analysis, we applied multiple linear regression for DT (values < 240 minutes) as outcome variable, quantile regression (Q25, Q50, Q75) for LOS, and Cox proportional-hazards model (right-censoring) along with non-parametric Kaplan-Meier estimator (log-rank test), for 1-year survival analysis. Final models were built using the stepwise method, selecting variables that had obtained differences in bivariate analysis. Estimated regression coefficients $(\beta)$ were considered significant with a $\mathrm{p}$-value $<0.05$. Odds and hazard ratios were expressed beside $95 \%$ confidence intervals (assumed valid without value $=1$ ). $R$ Project for Statistical Computing (3.5.2 version) software was used for data analysis.

\section{Results}

We analysed 192 episodes, after removing false positives $(n=47$; headache $17 \%$, 
seizures $13 \%$, dizziness $13 \%$, peripheral neuropathy $11 \%$, drugs $9 \%$, miscellanous $38 \%$ ).

The cohort was categorised (Figure 1, Table 1) into TRANSFER ( $\mathrm{n}=38$; $57.8 \%$ men) and NON-TRANSFER ( $\mathrm{n}=154 ; 51.9 \%$ women), who differed in age (67 vs. $81 ; \mathrm{p}<0.001)$, $\mathrm{BI}<60$ (2.6\% vs. $18 \%$; $\mathrm{p}=0.0268)$, mRankin $\leq 1$ (84\% vs. 67\%; $\mathrm{p}=0.0465)$, Oxfordshire/Bamford LACS/PACS/POCS/TACS topographic diagnosis ratio $(13 / 45 / 18 / 24$ vs. $38 / 41 / 7 / 14 ; \mathrm{p}=0.004), \mathrm{AF}+\mathrm{INR}<2$ (21\% vs. $13.6 \%$; $<<0.001)$, NIHSS (6 vs. $2 ; \mathrm{p}=0.0396)$, NIHSS $\geq 6+$ mRankin $\leq 1(42 \%$ vs. $5.8 \% ; \mathrm{p}=0.0102$ ), alteplase thrombolysis ( 9 vs. $7 ; \mathrm{p}=0.0008$ ), echocardiography ( $26 \%$ vs. $3.9 \%$; $\mathrm{p}=0.0118$ ). Only TRANSFER, according to NIHSS $\geq 6$, differed in MCT (71\% vs. $29 \%$; p < 0.001$)$ and SU (80\% vs. $20 \%$; p < 0.001). MT was performed in $50 \%$ of TRANSFER with NIHSS $\geq 6$. In evolutive analysis, there were differences in DT (146 vs. $396 \mathrm{~min}$; $\mathrm{p}<0.001$ ), discharge disposition ratio after hospital admission (home 60 vs. 78, death 11 vs. 2, subacute healthcare 29 vs $20 ; \mathrm{p}=0.01297$ ), LOS (6 vs. 1 days, $\mathrm{p}<0.001$ ), 30-days death in NIHSS $\geq 6+$ mRankin $\leq 1(10.5 \%$ vs. $0.6 \% ; \mathrm{p}=0.0057), 1$-year death NIHSS $\geq 6$ + mRankin $\leq 1(13.2 \%$ vs. $0.6 \% ; \mathrm{p}=0.0012)$ and 1 -year delta mRankin (3 vs. $1 ; \mathrm{p}$ $=0.0427$ ).

Regression models found an inverse relationship between NIHSS and DT $\left(\mathrm{R}^{2}\right.$ 0.3437; $\mathrm{p}<0.001$ ), although it seems linear only when NIHSS was above 5 points (Figure 2 and Figure 3 ); the strongest predictor of LOS was TACS $(\beta=3.14$ [0.03 - 8.49]; $\mathrm{p}=0.005$ ) (Table 2, Figure 4); and 1-year survival (Figure 5, Table 3) was related with TRANSFER (HR 4.68 [IC 95\% 1.37 - 16.07]; $\mathrm{p}=0.014$ ), age (HR 1.1 [IC 95\% 1.04 - 1.17]; p < 0.001), IB < 60 (HR 2.7 [IC 95\% 1.02 - 7.1]; p = 0.0447), TACS (HR 9.82 [IC 95\% 1.08 - 88.95]; $\mathrm{p}=0.0421$ ) and NIHSS $\geq 6$ (HR 2.85 [IC 95\% 1.05 - 7.74]; $\mathrm{p}=0.0401)$.

In TRANSFER + NIHSS $\geq 6$, MT $(n=10)$ was performed in $50 \%$ of cases and differences were found in MCT $(71 \%, \mathrm{p}=8 \mathrm{E}-5)$ in addition to SU admission $(80 \%, \mathrm{p}=7.77 \mathrm{E}-4)$.

\section{Discussion}

Transvictus study has allowed to describe the real-world attention of AIS/TIA patients from an unselected population, in a semi-rural community hospital.

False positives ratio was $20 \%$, similar to other published works, although greater than in the ischemic heart disease (10\% - 15\%) [12]. Without a doubt, the lack of advanced neuroradiology or neurovascular specialist represents a challenge for emergency physician's clinical judgement. Although risk stratification of large vessel occlusion is possible through predictive tools that use clinical semiology, the real-world praxis of ED is characterised by overcrowding and fear to err, what could lead to not recognising false negatives and asking the expert for an assessment too soon. Perhaps internal review of the most doubtful cases and clinical simulation using standardised algorithms could improve these assumptions. 


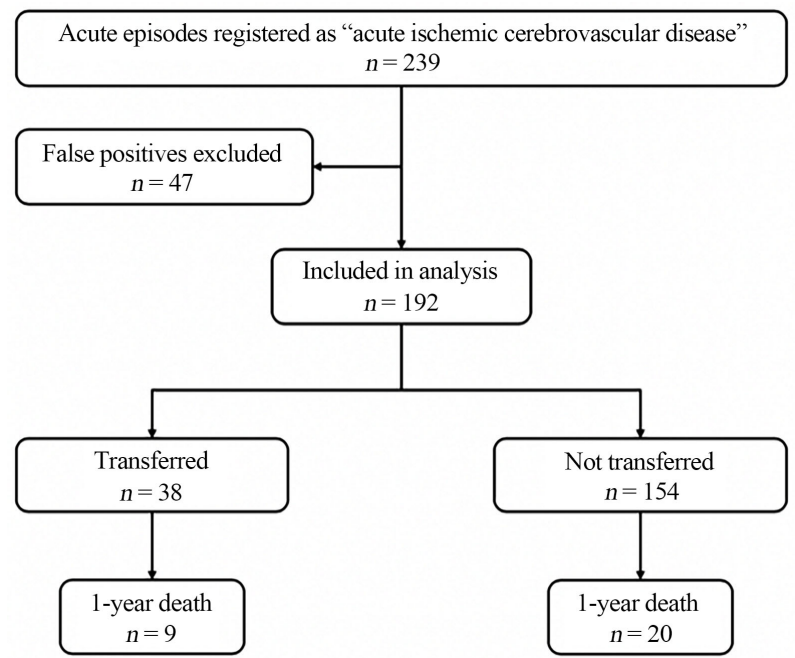

Figure 1. Flowchart of the study.
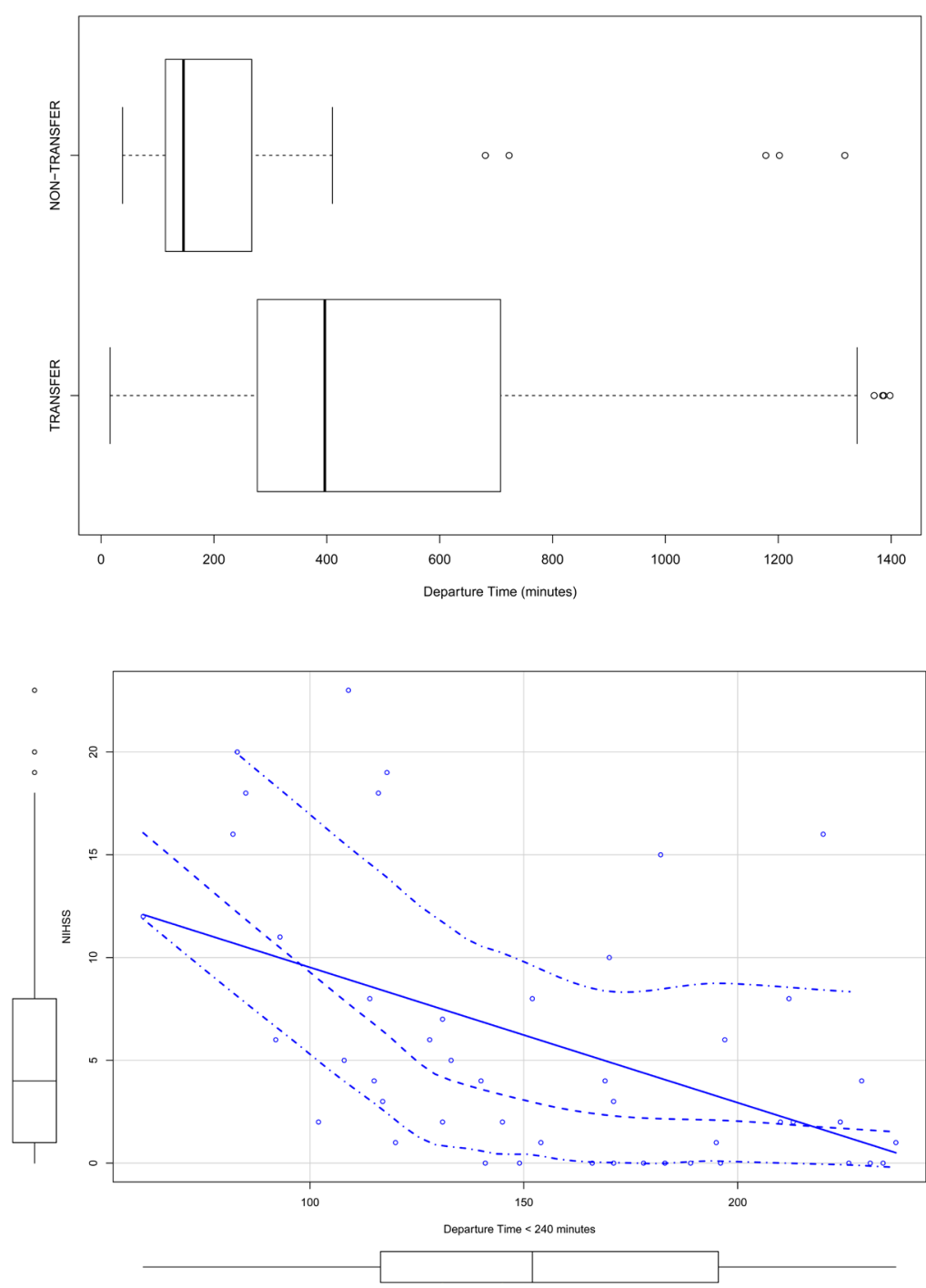

Figure 2. Analysis of Departure Time according to IFT decision and NIHSS. 

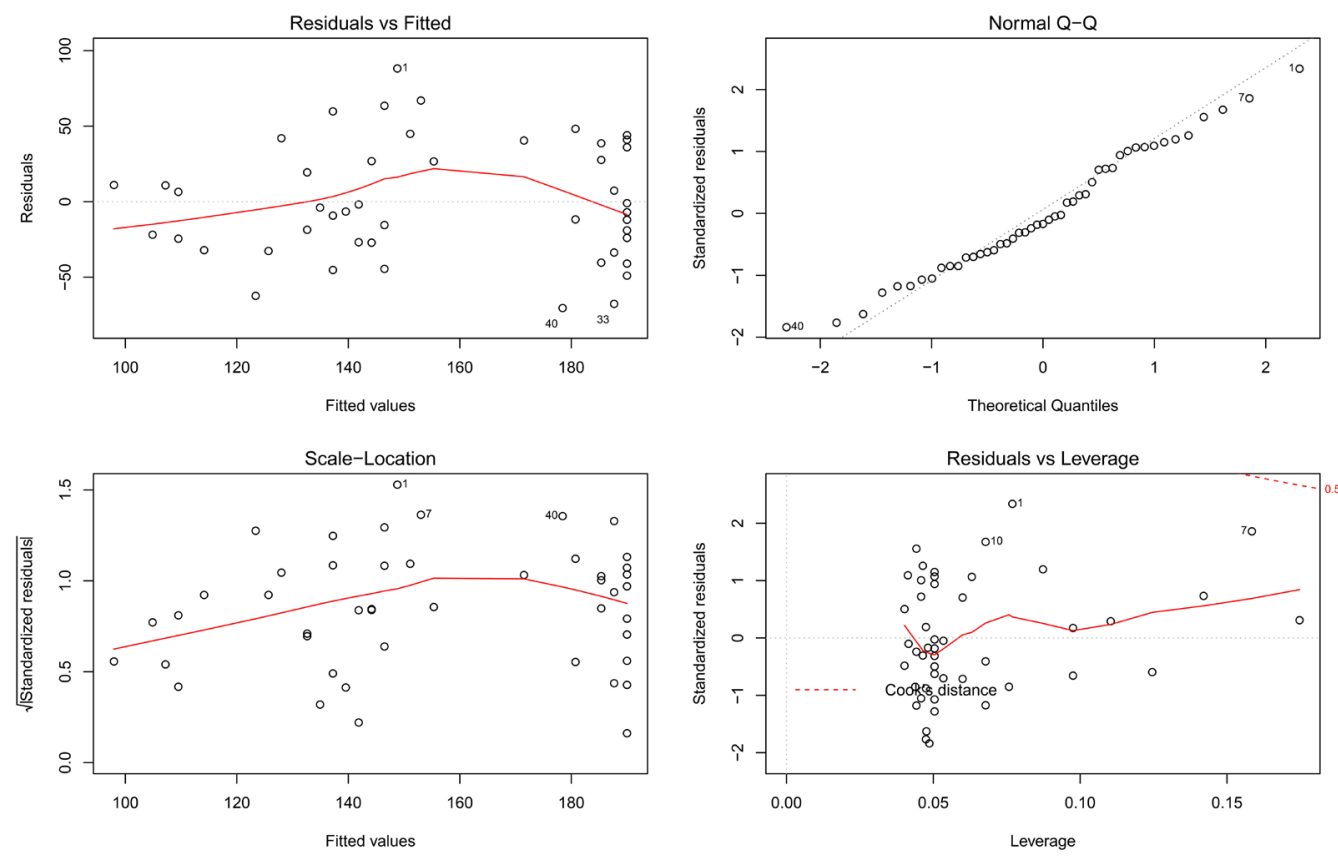

Figure 3. Simple regression for Departure Time (minutes). $\mathrm{R}^{2} 0.3437 ; \mathrm{p}=3.566 \mathrm{E}-05 . \mathrm{DT}=190-2.3 \times$ NIHSS $-38 \times$ TRANSF.
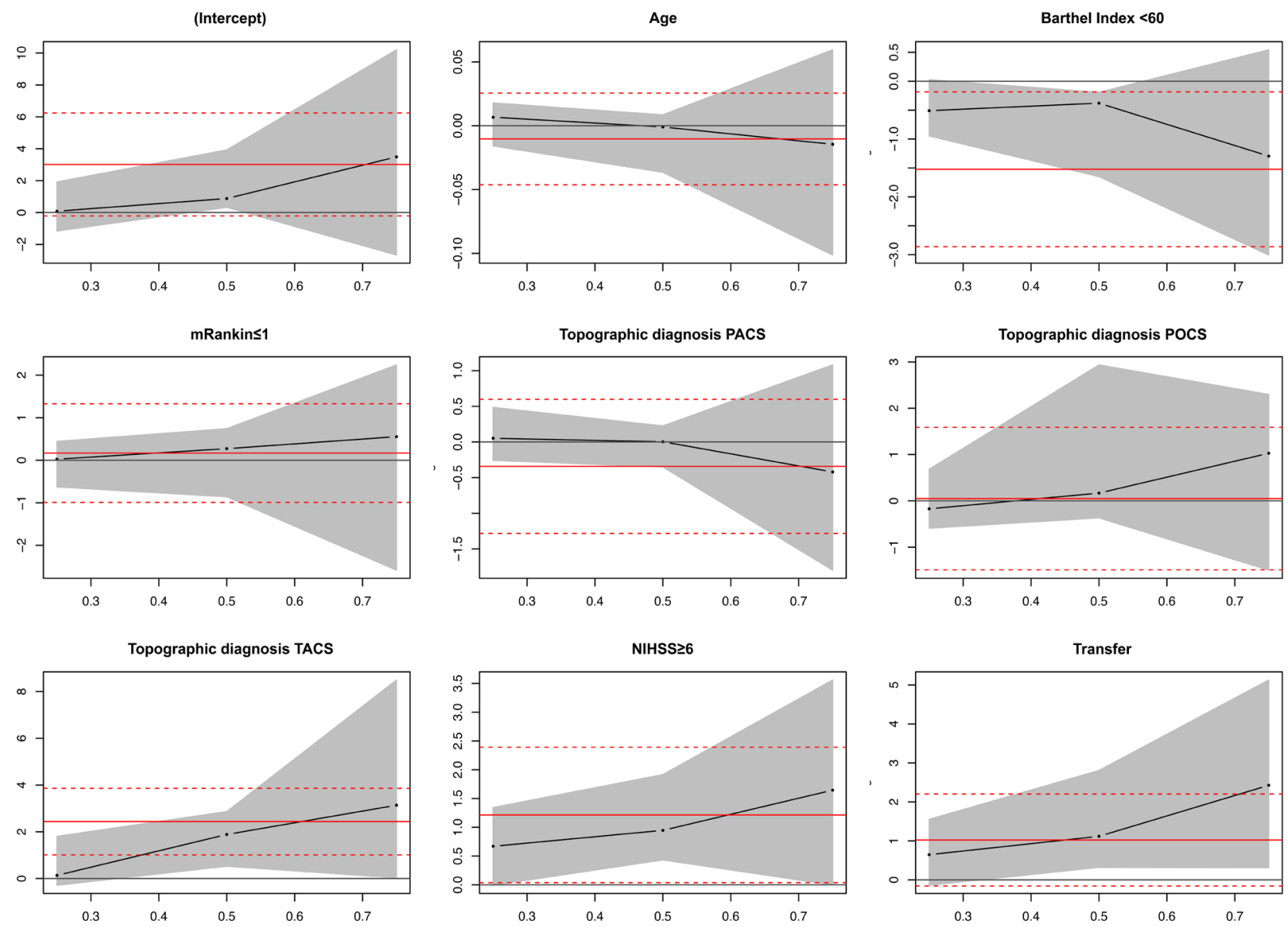

Figure 4. Plots of quantile regression for days of in-hospital stay. 


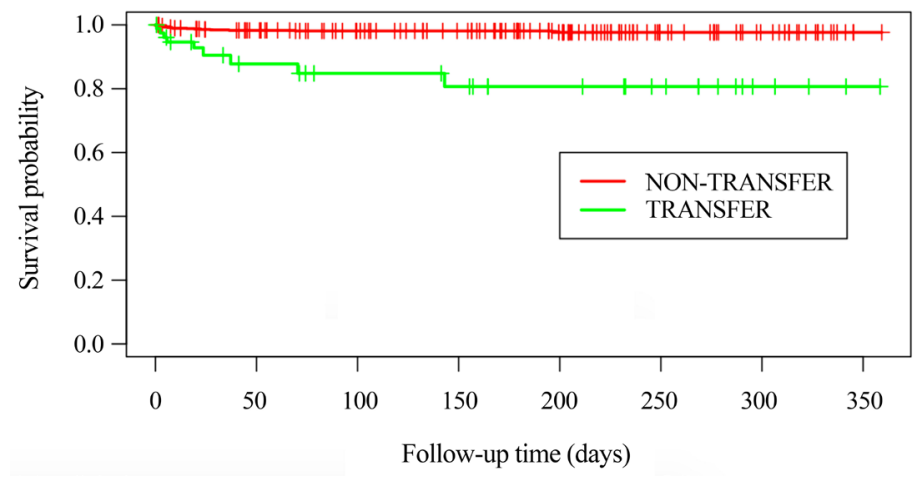

Figure 5. Kaplan-Meier survival analysis.

Table 1. Demographic and clinical characteristics of the cohort categorised according to IFT decision.

\begin{tabular}{|c|c|c|c|c|}
\hline & Total $(\mathrm{n}=192)$ & Transfer $(\mathrm{n}=38)$ & Non-Transfer $(\mathrm{n}=154)$ & $\mathrm{p}$ value \\
\hline \multicolumn{5}{|c|}{ Demographic Data } \\
\hline Age (median, IQR) & $80[66-86]$ & $67[60.5-80.75]$ & $81[68-87]$ & 0.0008 \\
\hline Gender (Men/Women) \% & $96 / 96$ & $22 / 16$ & $74 / 80$ & 0.3651 \\
\hline \multicolumn{5}{|c|}{ Functional Status and Medical History } \\
\hline Barthel Index (median, IQR) & $100[78.75-100]$ & $100[96.25-100]$ & $100[66.25-100]$ & 0.0193 \\
\hline Barthel Index $<60$ & 30 & 1 & 29 & 0.0268 \\
\hline mRankin (median, IQR) & $0[0-2]$ & $0[0-1]$ & $0[0-3]$ & 0.0284 \\
\hline mRankin $\leq 1$ & 135 & 32 & 103 & 0.0465 \\
\hline Charlson Index (mean, SD) & $4.45(2.14)$ & 3.89 & 4.58 & 0.0791 \\
\hline Smoke & 33 & 9 & 24 & 0.3809 \\
\hline Hypertension & 126 & 22 & 104 & 0.3526 \\
\hline Diabetes mellitus & 47 & 10 & 37 & 0.9336 \\
\hline Dyslipidemia & 74 & 16 & 58 & 0.7506 \\
\hline Cognitive impairment & 40 & 4 & 36 & 0.1275 \\
\hline Atrial fibrillation & 42 & 9 & 33 & 0.9345 \\
\hline Mitral valvulopathy & 22 & 3 & 19 & 0.5764 \\
\hline Peripheral arteriopathy & 13 & 0 & 13 & 0.0752 \\
\hline Ischemic cardiopathy & 18 & 3 & 15 & 0.9999 \\
\hline acetylsalicylic acid $100 \mathrm{mg}$ & 53 & 9 & 44 & 0.6884 \\
\hline acetylsalicylic acid $300 \mathrm{mg}$ & 9 & 1 & 8 & 0.6911 \\
\hline P2Y12 inhibitors & 14 & 1 & 13 & 0.3096 \\
\hline Vitamin $\mathrm{K}$ inhibitors & 22 & 5 & 17 & 0.7763 \\
\hline Direct oral anticoagulants & 7 & 3 & 4 & 0.1408 \\
\hline Angiotensin II converting enzyme inhibitors & 47 & 6 & 41 & 0.2378 \\
\hline Angiotensin II receptor antagonists & 19 & 5 & 14 & 0.5426 \\
\hline Lipid-lowering drugs & 60 & 13 & 47 & 0.8070 \\
\hline
\end{tabular}




\section{Continued}

\section{Emergency Department Management of Stroke}

\begin{tabular}{|c|c|c|c|c|}
\hline Own means, primary/prehospital/subacute healthcare $\%$ & $12 / 43 / 44 / 1$ & $10 / 29 / 60 / 0$ & $12 / 47 / 40 / 1$ & 0.1238 \\
\hline Topographic diagnosis (LACS, PACS, POCS, TACS, \%) & $33 / 42 / 9 / 16$ & $13 / 45 / 18 / 24$ & $38 / 41 / 7 / 14$ & 0.0041 \\
\hline Atrial fibrillation & 35 & 6 & 29 & 0.8412 \\
\hline International normalized ratio (median, IQR) & $1.06[1.01-1.14]$ & $1.07[1.04-1.18]$ & $1.06[1-1.13]$ & 0.2262 \\
\hline $\mathrm{AF}+\mathrm{INR}<2$ & 29 & 8 & 21 & $1.205 \mathrm{E}-5$ \\
\hline Time of onset of symptoms (hours) (median, IQR) & $2[1-4.5]$ & $2[1-4.5]$ & $2[1-4.5]$ & 0.7572 \\
\hline Wake-up stroke & 28 & 8 & 20 & 0.3228 \\
\hline NIHSS (median, IQR) & $2[0-6]$ & $6[2-15]$ & $2[0-4]$ & 0.039 \\
\hline NIHSS $\geq 6$ & 50 & 20 & 30 & $5.903 \mathrm{E}-16$ \\
\hline NIHSS $\geq 6+$ mRankin $\leq 1$ & 25 & 16 & 9 & 0.0102 \\
\hline Alteplase thrombolysis & 16 & 9 & 7 & 0.0008 \\
\hline Supra-aortic trunks ultrasound in TIA & 47 & 7 & 40 & 0.7279 \\
\hline Supra-aortic trunks ultrasound in AIS & 49 & 14 & 35 & 0.5959 \\
\hline Echocardiography & 16 & 10 & 6 & 0.0118 \\
\hline Magnetic resonance imaging & 18 & 8 & 10 & 0.4491 \\
\hline \multicolumn{5}{|c|}{ Clinical Evolution Data } \\
\hline Departure time from ED (minutes) (median, IQR) & $345[228-604]$ & $146[114-263]$ & $396[278-700]$ & $3.451 \mathrm{E}-5$ \\
\hline Disposition (home, death, subacute healthcare) \% & $75 / 4 / 21$ & $60 / 11 / 29$ & $78 / 2 / 20$ & 0.01297 \\
\hline Emergency Department reconsultation & 29 & 6 & 23 & 0.8628 \\
\hline Length of in-hospital stay (days) (median, IQR) & $1.59[0.70-4.92]$ & $6.03[3.29-10.55]$ & $1.06[0.59-3.22]$ & $3.146 \mathrm{E}-8$ \\
\hline 30-day death & 23 & 6 & 17 & 0.6206 \\
\hline 30-day death $($ NIHSS $\geq 6+$ mRankin $\leq 1$ ) & 5 & 4 & 1 & 0.0057 \\
\hline 1-year death & 29 & 9 & 20 & 0.2917 \\
\hline 1 -year death $($ NIHSS $\geq 6+$ mRankin $\leq 1$ ) & 6 & 5 & 1 & 0.0012 \\
\hline 1-year $\Delta$ Barthel Index (NIHSS $\geq 6$ ) & $10[0-30]$ & $0[0-22]$ & $22[0-41]$ & 0.2173 \\
\hline 1-year $\Delta$ mRankin (NIHSS $\geq 6$ ) & $2[1-3]$ & $3[1-5]$ & $1[1-3]$ & 0.0427 \\
\hline Median follow-up & $165.95[50.26-245.87]$ & $49.18[26.12-264.52]$ & $172.21[56.45-237.80]$ & 0.4885 \\
\hline
\end{tabular}

Table 2. Quantile regression for days of in-hospital stay (Min. 1, Q25 0.66, Q50 1.1292, Q75 3.8106, Max. 18).

\begin{tabular}{|c|c|c|c|c|c|c|}
\hline $\begin{array}{c}\text { Variable } \\
\text { Intercept }\end{array}$ & & $\beta$ Coefficient & \multicolumn{2}{|c|}{$95 \% \mathrm{CI}$} & $\mathrm{p}$ value & Analysis of Deviance \\
\hline & Q25 & 0.08617 & -1.15561 & 1.91956 & 0.91635 & \\
\hline & Q50 & 0.87711 & 0.32970 & 3.92495 & 0.15919 & \\
\hline & Q75 & 3.49057 & -2.66106 & 10.20243 & 0.16978 & \\
\hline \multirow[t]{4}{*}{ Age } & & & & & & 0.73931 \\
\hline & Q25 & 0.00669 & -0.01582 & 0.01786 & 0.46417 & \\
\hline & Q50 & -0.00095 & -0.03642 & 0.00852 & 0.89063 & \\
\hline & Q75 & -0.01451 & -0.10129 & 0.05950 & 0.60737 & \\
\hline
\end{tabular}




\section{Continued}

BI $<60$

0.09845

$\begin{array}{lllll}\text { Q25 } & -0.50928 & -0.94609 & 0.02577 & 0.13595 \\ \text { Q50 } & -0.37941 & -1.64902 & -0.19207 & 0.14232 \\ \text { Q75 } & -1.29452 & -3.00532 & 0.54322 & 0.21954\end{array}$

mRankin $\leq 1$

$\begin{array}{lllll}\text { Q25 } & 0.02500 & -0.62668 & 0.44286 & 0.93238 \\ \text { Q50 } & 0.27201 & -0.85739 & 0.74138 & 0.22372 \\ \text { Q75 } & 0.55417 & -2.58120 & 2.23950 & 0.54295\end{array}$

Oxford PACS

$\begin{array}{lllll}\text { Q25 } & 0.05196 & -0.25704 & 0.48497 & 0.82800 \\ \text { Q50 } & 0.00291 & -0.34938 & 0.22445 & 0.98716 \\ \text { Q75 } & -0.42039 & -1.79741 & 1.08054 & 0.56960\end{array}$

Oxford POCS

$\begin{array}{ll}\text { Q25 } & -0.17254 \\ \text { Q50 } & 0.16744 \\ \text { Q75 } & 1.02749\end{array}$

Oxford TACS

$\begin{array}{ll}\text { Q25 } & 0.13516 \\ \text { Q50 } & 1.88513 \\ \text { Q75 } & 3.13684\end{array}$

$$
\begin{aligned}
& -0.59061 \\
& -0.36747
\end{aligned}
$$

0.68173

2.93412

2.29686

0.70787

$-1.49540$

.

0.04320

$\begin{array}{ll}-0.28569 & 1.80260 \\ 0.52886 & 2.86266 \\ 0.03303 & 8.49063\end{array}$

0.70895

$<0.001$

0.00557

NIHSS $\geq 6$

$\begin{array}{ll}\text { Q25 } & 0.66951 \\ \text { Q50 } & 0.94644 \\ \text { Q75 } & 1.64514\end{array}$

$-0.00717$

1.34282

0.43326

1.91522

$-0.00208$

3.55895

0.33732

Transfer

$\begin{array}{lllll}\text { Q25 } & 0.64545 & -0.13834 & 1.55109 & \mathbf{0 . 0 3 2 7 5} \\ \text { Q50 } & 1.11639 & 0.32021 & 2.80571 & <0.001 \\ \text { Q75 } & 2.42533 & 0.31387 & 5.12561 & \mathbf{0 . 0 0 9 6 6}\end{array}$

Table 3. Cox regression for 1-year survival after ED visit.

\begin{tabular}{ccccc}
\hline Variable & $\beta$ Coefficient & HR & $95 \%$ CI & p value \\
\hline Age & 0.0999 & 1.1 & $1.04-1.17$ & $\mathbf{0 . 0 0 0 8 0 1}$ \\
BI $<60$ & 0.9919 & 2.7 & $1.02-7.1$ & $\mathbf{0 . 0 4 4 6 9 4}$ \\
mRankin $\leq 1$ & -0.9103 & 0.4 & $0.14-1.2$ & 0.100278 \\
Oxford PACS & 0.9398 & 2.56 & $0.29-22.23$ & 0.394218 \\
Oxford POCS & 2.008 & 7.45 & $0.63-88.59$ & 0.111871 \\
Oxford TACS & 2.2846 & 9.82 & $1.08-88.95$ & $\mathbf{0 . 0 4 2 1 5 0}$ \\
NIHSS $\geq 6$ & 1.0472 & 2.85 & $1.05-7.74$ & 0.040087 \\
DT & -0.0008 & 1 & $0.99-1$ & 0.298260 \\
\hline
\end{tabular}


Patients with worse severity and better basal functional status were transferred, which is consistent with the recommendations of the clinical practice guidelines. However, the MCT allowed half of those transferred to be rejected for TM due to false positives, lacunar syndromes or successful reperfusion after alteplase administration. One thing that perhaps would have predicted this situation is that some of the patients with a worse initial NIHSS experienced early improvement, regardless of alteplase administration, and yet they were transferred anyway, which is also consistent with the results of other authors [13].

IFT to complement risk stratification of TIA with SAT was observed in non neglegible proportion (18\%), and one of them was admitted in SU to perform carotid neurosurgery. This group of patients would probably be reduced if emergency physicians had been trained in basic neurosonology.

Severity (NIHSS) and celerity in decision making (DT) were related when adjusted according to TRANSFER (analysed DT $<4$ hours). Optimisation of the functional strategy in the emergency department (early location of the patient in the examination and CT rooms, laboratory response time and periodic teleictus simulation exercises) should be an element to be considered [14]. mRankin $\leq 1$ predicted worse DT, which could be explained by co-linearity with the lowest NIHSS, or TIA patients, which were observed a longer time before final disposition. LOS was shorter for non-transfer, something explainable because the goal of admission to an acute center was primarily to ensure their clinical stability, and transfer them to a subacute healthcare center in order to optimize their functional status. In contrast, transfer received many complementary examinations or invasive techniques in the acute center, which prolonged LOS.

Survival analysis showed that the prognosis of transfer was worse. This finding is very relevant for emergency medicine, as all efforts should focus on reducing the proportion of false negatives, especially of TACS, and/or higher NIHSS.

The limitations of the study are the unicentric design and the retrospective analysis, which could have caused the loss of some patients registered with an incorrect code in our computer system.

\section{Conclusion}

Transvictus study has allowed us to know how AIS/TIA patients are attended in the ED of a semi-rural community hospital, and the clinical process of the decision to transfer to a highly complex center. Shared decision-making with a stroke unit through telemedicine facilitated a more standardised clinical management in our non-metropolitan setting, although we identified several improvement opportunities: first, obtaining an MCT when considering an IFT, especially for the most uncertain cases; second, DT optimization of transferred patients through functional changes in ED; and thirdly, training of emergency physicians in basic neurosonology, which could improve risk stratification of TIA and therefore avoid some transfers for this reason alone. 


\section{Conflicts of Interest}

The authors declare no conflicts of interest regarding the publication of this paper.

\section{References}

[1] Ministry of Health, Consumer Affairs and Social Welfare (2019). Specialized Care Registry (RAE-CMBD)-Main Diagnoses. Madrid. https://pestadistico.inteligenciadegestion.mscbs.es/

[2] National Institute of Statistics (2019) Hospital Morbidity Survey-Year 2017. Madrid. http://www.ine.es/

[3] Catalan Agency for Healthcare Quality and Assessment (AQuAS) (2019) Codi Ictus Catalunya Register (CICAT). Barcelona. http://aquas.gencat.cat/

[4] George, B.P., Doyle, S.J., Albert, G.P., Busza, A., Holloway, R.G., Sheth, K.N., et al. (2018) Interfacility Transfers for US Ischemic Stroke and TIA, 2006-2014. Neurology, 90, e1561-e1569. https://doi.org/10.1212/WNL.0000000000005419

[5] Sonig, A., Lin, N., Krishna, C., Natarajan, S.K., Mokin, M., Hopkins, L.N., et al. (2016) Impact of Transfer Status on Hospitalization Cost and Discharge Disposition for Acute Ischemic Stroke across the US. Journal of Neurosurgery, 124, 1228-1237. https://doi.org/10.3171/2015.4.JNS141631

[6] Demaerschalk, B.M., Berg, J., Chong, B.W., et al. (2017) American Telemedicine Association: Telestroke Guidelines. Telemedicine Journal and e-Health, 23, 376-389. https://doi.org/10.1089/tmj.2017.0006

[7] Merino, J.G., Luby, M., Benson, R., Davis, L.A., Hsia, A.W., Latour, L.L., et al. (2013) Predictors of Acute Stroke Mimics in 8,187 Patients Referred to a Stroke Service. Journal of Stroke and Cerebrovascular Diseases, 22, e397-e403. https://doi.org/10.1016/j.jstrokecerebrovasdis.2013.04.018

[8] Kiyohara, T., Kamouchi, M., Kumai, Y., Ninomiya, T., Hata, J., Yoshimura, S., et al. (2014) ABCD3 and ABCD3-I Scores Are Superior to ABCD2 Score in the Prediction of Short- and Long-Term Risks of Stroke after Transient Ischemic Attack. Stroke, 45, 418-425. https://doi.org/10.1161/STROKEAHA.113.003077

[9] Saedon, M., Hutchinson, C.E., Imray, C.H.E. and Singer, D.R.J. (2017) ABCD2 Risk Score Does Not Predict the Presence of Cerebral Microemboli in Patients with Hyper-Acute Symptomatic Critical Carotid Artery Stenosis. Stroke and Vascular Neurology, 2, 41-46. https://doi.org/10.1136/svn-2017-000073

[10] Ottaviani, M., Vanni, S., Moroni, F., Peiman, N., Boddi, M. and Grifoni, S. (2016) Urgent Carotid Duplex and Head Computed Tomography versus ABCD2 Score for Risk Stratification of Patients with Transient Ischemic Attack. European Journal of Emergency Medicine, 23, 19-23. https://doi.org/10.1097/MEJ.0000000000000165

[11] National Institute for Health and Care Excellence (2019) Transient Ischaemic Attack. NICE Pathways, London. https://pathways.nice.org.uk/pathways/stroke/transient-ischaemic-attack

[12] Regueiro, A., Fernandez-Rodriguez, D., Freixa, X., Bosch, X., Martin-Yuste, V., Brugaletta, S., et al. (2018) Falsos positivos en la activacion por IAMCEST en una red regional: Analisis integral e impacto clinico. Resultados del registro Codi Infart de Cataluna [False Positive STEMI Activations in a Regional Network: Comprehensive Analysis and Clinical Impact. Results from the Catalonian Codi Infart Network]. Revista Espanola de Cardiologia, 71, 243-249.

https://doi.org/10.1016/j.recesp.2017.05.025 
[13] Zachrison, K.S., Leslie-Mazwi, T.M., Boulouis, G., Goldstein, J.N., Regenhardt, R.W., Viswanathan, A., et al. (2019) Frequency of Early Rapid Improvement in Stroke Severity during Interfacility Transfer. Neurology Clinical Practice, 9, 373-380. https://doi.org/10.1212/CPJ.0000000000000667

[14] Hayes, M., Schlundt, D., Bonnet, K., Vogus, T.J., Kripalani, S., Froehler, M.T., et al. (2019) Tales from the Trips: A Qualitative Study of Timely Recognition, Treatment, and Transfer of Emergency Department Patients with Acute Ischemic Stroke. Journal of Stroke and Cerebrovascular Diseases, 28, 1219-1228.

https://doi.org/10.1016/j.jstrokecerebrovasdis.2019.01.012 